\title{
An Investigation on the Feasibility of Simulating the Distillation Curves and ASTM Distillation Temperature
}

\author{
Khalid Farhod Chasib ${ }^{1,}$, , Srikanth Karthik P. \\ ${ }^{1}$ Head of the Petroleum \& Gas Engineering Department, Collage of Engineering, University of Thi Qar, Thi Qar, Iraq \\ ${ }^{2}$ Visakh Refinery, Hindustan Petroleum Corporation Limited, Visakhapatnam, India
}

Email address:

khalid_farhod@utq.edu.iq (Khalid F. C.), pskarthikiitb@gmail.com (Srikanth K. P.)

${ }^{*}$ Corresponding author

\section{To cite this article:}

Khalid Farhod Chasib, Srikanth Karthik P. An Investigation on the Feasibility of Simulating the Distillation Curves and ASTM Distillation Temperature. International Journal of Oil, Gas and Coal Engineering. Vol. 5, No. 5, 2017, pp. 80-89. doi: 10.11648/j.ogce.20170505.13

Received: October 31, 2016; Accepted: December 22, 2016; Published: October 24, 2017

\begin{abstract}
For the reliable design of crude oil and residua fractionators and fired heaters, accurate calculation method for ASTM Distillation curves and ASTM distillation temperature at the required volume percentage distilled is necessary. The present work deals with fitting literature data of ASTM Distillation curves and obtain a new correlation suitable to use in modeling when optimizing the refining processes, such as distillation, FCC, catalytic reforming, hydrotreating, etc. The results showed that the values predicted by the new correlation are in very close agreement with literature data and the range of mean overall deviation (\%MOD) is $(0.305-0.585)$. Also a shortcut correlation is presented to calculate the ASTM distillation temperature at the required volume percentage distilled. Based on temperatures obtained we can have an estimate of the heating costs involved to perform distillation and temperature at which different cuts are obtained.
\end{abstract}

Keywords: Distillation Curves, ASTM, Refining Processes

\section{Introduction}

Most of the mathematical models used for representing any type of phenomena (or situation) occurring not only during chemical reactions but also in other areas, e.g., thermodynamics, environmental sciences, molecular modeling, etc. involve parameters that need to be estimated from experimental data. The models can be supported on theoretical, semitheoretical/ semiempirical or empirical bases, and their parameters can have theoretical meaning or be simply correlation constants.

Distillation curves provide a breadth of information about the crude oil or the petroleum fuel. In certain respects, the boiling point distribution is representative of the composition of the petroleum fraction. Therefore, in principle, by determining the presence and volume percent of the components in a conventional hydrocarbon fuel, the overall physical properties can be determined [1].

The simple distillation curve is the temperature as a function of the percent distilled in a simple or Rayleigh type of distillation. This type of distillation is approximated by the laboratory A.S.T.M. distillation which is widely used to characterize petroleum fractions. The A.S.T.M. procedure gives some reflux and rectification, and the results are not exactly equal to the simple batch distillation, although the difference is not large. The temperature normally measured is the condensation temperature of the vapor flowing from the still to the condenser [1].

There are many types of standard distillation tests that determine the boiling point distribution of petroleum fuels, the inter-conversion between which is well documented. Some of the more common standard test methods for distillation of petroleum products include: ASTM D86-96, which is performed under atmospheric pressure and is used for determining the boiling point distribution of light petroleum fractions, such as naphtha, kerosene, diesel, and light gas oil; micro-distillation; molecular distillation; fractional distillation (typically using a spinning band still); ASTM D5236 distillation (typically using a pot still); D1160 (for heavy petroleum fractions); ASTM D3710 (simulated distillation, which is also known as the GC SimDist method, and uses gas chromatography to determine the true boiling point, or TBP, of gasoline); ASTM D2887 (GC SimDist to 
determine the TBP of petroleum fraction other than gasoline); ASTM D2892 (also known as 15/5 distillation, which produces simulated TBP of petroleum fuels using a distillation column with 15 theoretical plates and a reflux ratio of 5); ASTM D5236 Distillation (also known as the vacuum pot still method, and is used for heavy hydrocarbon mixtures); ASTM D5307 (SimDist for determining TBP of crude oil); ASTM D6352-98; and Hemple analysis for the distillation of a large volume of fuel samples providing further detailed analysis of the produced distilled cuts. ASTM D86-96 and D1160 may be combined together for determining the boiling point distribution of wide boiling range materials, such as crude oils [2].

ASTM Distillation tests for gasoline, naphtha, and kerosene (D86); natural gasoline (D216); and gas oil (D158) involve much the same procedure. A somewhat similar test known as the "Engler distillation" has been used in the past, and often the ASTM distillation is referred to as "an Engler" [2].

The ASTM distillation curve is conducted on the whole crude. This type of distillation curve is used however on a routine basis for plant and product quality control. This test is carried out on crude oil fractions using a simple apparatus designed to boil the test liquid and to condense the vapors as they are produced. Vapor temperatures are noted as the distillation proceeds and are plotted against the distillate recovered. Because only one equilibrium stage is used and no reflux is returned, the separation of components is poor and mixtures are distilled [3].

The molecular distillation process $[4,5]$ introduces a potential technique for attainment of liquid volume percentage in relation to temperature for the distilled fractions. In Batistella [6], the robustness of this method can be verified since it enables operation at low temperatures, short residence times, being ideal for working with high molecular weight and thermally sensitive compounds [7]. In the petroleum case, it avoids thermal cracking, allowing thus, the development of a methodology for determination of the real TBP curve. It is necessary however, to establish a relationship between the operating conditions of the molecular distiller and the TBP curve $[8,9]$. In Batistella et al. [10], data of temperature and percentage of distillate from molecular distiller are obtained experimentally, were used in the TBP curve extension, and a new correlation (FRAMOL correlation) was presented [11]:

$$
T B P=456.4+0.1677 \times T_{D M}+1.64 \cdot 10^{-4} \times T_{D M}^{2}+4.13 \cdot 10^{-6} \times T_{D M}^{3}
$$

where: $T B P=$ True Boiling Point $\left({ }^{\circ} \mathrm{C}\right)$;

$T_{D M}=$ Operating temperature of the Molecular Distiller $\left({ }^{\circ} \mathrm{C}\right)$.

The main objective of the present work is to fit literature data of ASTM Distillation curves and obtain a new correlation suitable to use in modeling when optimizing the refining processes, such as distillation, FCC, catalytic reforming, hydrotreating, etc. Also a shortcut correlation is presented to calculate the ASTM distillation temperature at the required volume percentage distilled.

\section{Fitting of ASTM Distillation Data}

Distillation data and specific gravity are the most common properties used as inputs in empirical correlations to characterize petroleum fractions. This characterization is achieved by means of correlations that are useful for determining molecular weight, critical properties, and so forth. They can also be utilized for distinguishing reaction products as pseudocomponents or lumps (naphtha, middle distillates, etc.) of some typical refinery processes such as hydrocracking, catalytic cracking, and so forth. To have accurate and reliable representations of distillation data for further interpolation, a strict analysis of other approaches apart from the traditional interpolation techniques is mandatory [4-5].

In order to develop a correlation for certain thermodynamic property, one has to determine all the parameters that influence this property and then studies the influence of each of these parameters on the property under consideration, using literature data available for this purpose [12].

In the past five decades the researchers tried to calculate the properties of crude oil or the petroleum fuel by making a mathematical model, which is a function of composition and some constants, which fits the experimental data. This mathematical model is not supported by any theoretical base. With the development of computer and computer programs the use of analytical expressions to interpolate, extrapolate and even predict thermodynamic information has become of increasing importance for process design and for modeling of process operation [13].

ASTM Distillation curves of refining fractions can be adequately described by an empirical equation. The equation is suitable for insertion in models used for optimizing operation of refining processes such as distillation, FCC, catalytic reforming, hydrotreating, etc. the equation takes the form of:

$$
V=\left\{1-e^{-\left(\frac{\Psi}{\alpha}\right)^{\beta}}\right\} \times 100
$$

where $V$ is the volume percent distilled at temperature $T$ and $\alpha$ and $\beta$ are constants and determined from the data of ASTM distillation curve, $\Psi$ is the dimensionless temperature defined as:

$$
\Psi=\frac{T-T_{i}}{T_{f}-T_{i}}
$$

where $T_{i}$ and $T_{f}$ are the initial and final boiling point temperatures of ASTM distillation respectively. By defining dimensionless temperature $\Psi$, the constant of Eq. 2 are invariant with respect to unit of temperature. 


\section{Short-Cut Correlation for Calculating ASTM Distillation Temperature}

Many a times, petroleum refinery engineering is taught in the undergraduate as well as graduate education in a technological but not process design perspective. While technological perspective is essential for a basic understanding of the complex refinery processes, a design based perspective is essential to develop a greater insight with respect to the physics of various processes, as design based evaluation procedures enable a successful correlation between fixed and operating costs and associated profits. In other words, a refinery engineer is bestowed with greater levels of confidence in his duties with mastery in the subject of refinery process design [1].

A process design engineer is bound to learn about the basic knowledge with respect to a simulation problem and its contextual variation with a process design problem. A typical process design problem involves the evaluation of design parameters for a given process conditions. However, on the other hand, a typical process simulation problem involves the evaluation of output variables as a function of input variables and design parameters. One of the important features of refinery process design is to estimate the crude distillation product properties (TBP) for a given assay of the crude oil [2].

Empirical correlations are also very popular to estimate "bulk" properties of petroleum fractions, which are mainly based on distillation curves and specific gravities. These correlations are very useful in process engineering, particularly when the available experimental data are limited. In the case of distillation curves, sometimes a limited amount of distillation points are available and it is necessary to interpolate/extrapolate to determine a required value. Several American Society for Testing and Materials (ASTM) methods are commonly used to obtain distillation data: ASTM D-5307, ASTM D-2892, ASTM D-1160, ASTM D-86, and so forth. All of them employ standardized devices and report boiling point temperatures of the sample versus distillation yields on a volumetric and/or a gravimetric basis. Since distillation curves are formed with a finite number of temperature-yield data, they can be fitted to different functions in order to generate a continuous representation. Polynomial regression, cubic spline interpolation, and Lagrange interpolation are all common mathematical tools which have been used for interpolating points along the distillation curve. Another approach, which offers more accurate adjustments, is the use of least-square methods for fitting probability distribution functions to distillation data [4-5].

A new short-cut correlation is developed for fitting data of ASTM distillation data curves. Equation 2 is rearranged to calculate the ASTM distillation Temperature at the required volume percentage distilled. The equation takes the form of:

$$
T=\left\{\left(T_{50}-T_{i}\right) \times\left(\frac{\alpha}{\Psi}\right) \times e e^{\left.\left[\frac{\left.\ln -\ln 1-\frac{V}{100}\right)}{\beta}\right]\right]}\right]+T_{i}
$$

where $\mathrm{V}$ is the Volume percent distilled at temperature $\mathrm{T}, \mathrm{T}_{\mathrm{i}}$ is the initial boiling Temperature of ASTM distillation and $\mathrm{T}_{50}$ is the boiling Temperature of ASTM distillation at $50 \%$ of volume distilled.

\section{Maximum-Likelihood Principle}

The estimation of parameters in theoretical and semiempirical mathematical models from experimental data is an important requirement in many fields of science and engineering. In the maximum-likelihood analysis, it is assumed that all measured data are subject to random errors. If each experiment were replicated, the average value for each replicated experimental point would approach some true value. Usually the distribution of a measured variable about its true value is approximated by the normal distribution, characterized by an associated variance. The variances are ideally obtained from replicated experiments, but they may be estimated from experience associated with a particular type of experimental apparatus. It is customary to assume that the random errors in different experiments are uncorrelated [14].

For each experiment, the true values of the measured variables are related by one or more constraints. Because the number of data points exceeds the number of parameters to be estimated, all constraint equations are not exactly satisfied for all experimental measurements. Exact agreement between theory and experiment is not achieved due to random and systematic errors in the data and to "lack of fit" of the model to the data. Optimum parameters and true values corresponding to the experimental measurements must be found by satisfaction of an appropriate statistical criterion [14].

If this criterion is based on the maximum-likelihood principle, it leads to those parameter values that make the experimental observations appear most likely when taken as a whole. The likelihood function is defined as the joint probability of the observed values of the variables for any set of true values of the variables, model parameters, and error variances. The best estimates of the model parameters and of the true values of the measured variables are those which maximize this likelihood function with a normal distribution assumed for the experimental errors [14].

The parameter estimation algorithm based on the maximum likelihood principle, converges rapidly for almost any initial estimates of the parameters. The rapid convergence is due in part to the similarity to Gauss-Newton method and in part to the successful application of a step-limiting procedure that assures superior convergence behavior [15].

The maximum likelihood principle method provides a mathematical and computational guarantee of global optimality in parameter estimation that provides the best fit to measured data [15]. The objective function in nonlinear parameter estimation problems is given by equation (5).

\section{Determination of Constants}

Constants $\alpha$ and $\beta$ are calculated such that Eq. 2 fits the literature data $[1,2,13,16-20]$ of ASTM distillation. The 
properties of the crude oils and petroleum fractions used are shown in Table 1. To obtain the best fitting that describe the ASTM curves, the sum of square of errors for prediction should be minimum. Let $T_{1}, T_{2}, \ldots, T_{n}$ and $V_{1}, V_{2}, \ldots, V_{n}$ be the data of ASTM distillation test. The values of $\alpha$ and $\beta$ should minimize the following function:

$$
F=\sum_{i=1}^{n}\left[\left\{1-e^{-\left(\frac{\Psi}{\alpha}\right)^{\beta}}\right\} \times 100-V_{i}\right]^{2}
$$

The problem of minimization is non-linear optimization, and an analytical solution is difficult to obtain. A numerical method, employing the maximum-likelihood principle technique, has been developed to resolve the above problem. In this numerical method, the function $F$ is minimized with respect to $\alpha$ and $\beta$. The procedure of the numerical method is complex and is not discussed here.

A computer program was designed and developed by using Matlab software for the purpose of this work. The basic design structure involves modularized procedures or subroutines to facilitate testing and development. The whole structure of the computer program was subjected to numerous tests and debugging procedures, and was only completed after undergoing many developments to optimize calculation procedures. The flow chart of computer program illustrated schematically in figure 1.

Using the data from literature $[1,2,13,16-20]$, the values of $\alpha, \beta$ and $\alpha / \Psi$ has been determined, and can be used as universal constants.

$$
\frac{\alpha}{\Psi}=1.11
$$

$\alpha=0.6$

$\beta=3.49$

Hence equation (4) takes the form:

$$
T=\left\{1.11 \times\left(T_{50}-T_{i}\right) \times e^{\left[\frac{\ln \left(-\ln \left(1-\frac{V}{100}\right)\right.}{3.49}\right]}\right]+T_{i}
$$

Validity range, $\mathrm{V}=25 \%$ to $95 \%$

The above equation matches closely with experimental data (around 90 to $95 \%$ ). The shortcut correlation is a simple, but remarkably accurate, formula for the $\%$ volume distilled dependence on temperature. With a simple pocket calculator we can now calculate the required temperature.

\section{Statistical Measurement and Analysis of Dispersion}

The applicability and accuracy of any proposed correlation it is very important to know how this correlation fits the literature data which is done by comparing the obtained results from the proposed correlation with the literature data.

The various measurement of dispersion or variation are available, the most common being the Mean Overall Deviation.

The percentage of Mean Overall Deviation " $\%$ MOD" is a more tangible element indicating the overall goodness of the fit of the data by the correlation and it defines as [14]:

$$
\% M O D=\frac{\sum_{i=1}^{n}\left|\frac{M_{i}^{\text {lit. }}-M_{i}^{\text {calcd }}}{M_{i}^{\text {lit. }}}\right|}{n} \times 100
$$

where $M$ is an intensive property and $n$ is the number of literature data point.

\section{Results and Discussion}

Petroleum is evaluated mainly in terms of its ASTM distillation curve, what makes possible to investigate the yields of the products that will be obtained in the refineries, as well as to establish operational strategies and process optimization.

ASTM distillation curves are drawn for each blend component, with the temperature on the vertical axis ( $\mathrm{Y}$-axis) and the volume percent distilled on the horizontal axis (X-axis). Distillation must be on a consistent basis for all components; that is, either percent evaporated or percent recovered.

The temperature at any point is the averaged result of a large number of components and includes all the effects of non-ideality in the solutions. Thus in most cases it is impossible to relate such a curve to the volatility of the individual components involved. As a result, such simple distillation curves are not of much direct value for the solution of rectification problems.

A direct and universal approach that can be a guarantee of the best solution during parameter estimation process is not easy to develop. The main difficulties when estimating parameters in heterogeneous kinetics are: the complexity of the model, which can be from simple algebraic equations to complex differential equation systems, linear or highly nonlinear in nature; the source and precision of experimental data, which can come from the literature (from one or more references) or from own or literature experiments specially designed to perform kinetic studies in which all the care has been put to assure kinetic regime; the robustness of the optimization algorithm.

Model selection should be based not solely on goodness of fit but also on the degree of confidence of the predicted parameters. It is well-known that increasing the number of free parameters to be estimated can improve the goodness of fit but can also decrease the confidence in the estimates of the model parameters.

As shown in Figures 2 to 7, the numerical method successfully fits Eq. 2 to ASTM distillation data of the feed and products of FCC unit and for Crude Oil. The Mean Overall Deviation "mean D\%" for all ASTM distillation curves is very small which reflect the agreement between prediction and literature data as illustrated in table 2 .

Validating the Eq. 6 with ASTM Distillation curve data 
available in literature [19-21] are shown in Figures 8 and 9. The errors during data fitting were obtained with boiling points close to the end of the distillation curves followed by those close to the beginning. This problem was particularly evident for the normal and Student's t distribution functions, which are symmetrical. The difficulty in fitting distribution functions to IBP and FBP data is compounded by the larger experimental error associated with the endpoints of distillation curves. These errors are associated with the sensitivity of experimental devices when initializing or finalizing the tests and are observed regardless of whether the equipment is operated manually or automatically. The experimental error is variable, depending upon the standardized method that is employed; for instance, in the ASTM D-2892 method, errors up to $1.2 \mathrm{wt} \%$ for the volume recovery are tolerated, whereas in the ASTM D-1160 method, errors can range from 1.7 up to $5.7 \mathrm{wt} \%$ for the different points in the distillation curve.

\section{Conclusions}

The properties of natural petroleum and petroleum products make use of the ASTM distillation analyses very useful for petroleum characterization, design and operation of refinery units, the classification of petroleum, the development of petroleum property correlations and it has been used worldwide.

In this work different literature data have been used to validate the correlation. Optimum values of constants $\alpha$ and $\beta$ are significantly different for each case (Figures 2 to 7 ), using the enormous data from literature these value can be used as universal constant as illustrated in table 3. Eq. 2 has been successfully integrated in model of FCC Unit for blending ASTM distillation curves of fresh feed, recycle $\mathrm{LCO}$ and recycle $\mathrm{HCO}$ for the total feed to the reactor.

Regarding the usage of the shortcut equation (Eq. 6), if we know IBP and boiling point temperature at $50 \%$ of volume distilled, we can calculate the temperature for the range of $25 \%$ volume distilled to $95 \%$ volume distilled. Based on temperatures obtained we can have an estimate of the heating costs involved to perform distillation and temperature at which different cuts are obtained.

\section{Nomenclature}

\section{Abbreviations}

$\begin{array}{ll}\text { ASTM } & \text { American Society for Testing Materials } \\ \text { FCC } & \text { Fluidized catalytic Cracking } \\ \text { HCO } & \text { Heavy Cycle Oil } \\ \text { IBP } & \text { Initial Boiling Point }\left({ }^{\circ} \mathrm{C}\right) \\ \text { LCO } & \text { Light Cycle Oil } \\ \text { MOD } & \text { Mean Overall Deviation } \\ \text { TBP } & \text { True Boiling Point }\left({ }^{\circ} \mathrm{C}\right)\end{array}$

\section{Symbols}

\section{Greek Symbols}

$\begin{array}{ll}\alpha & \text { Constant of new Correlation (-) } \\ \beta & \text { Constant of new Correlation (-) } \\ \psi & \text { Dimensionless Temperature (-) }\end{array}$

\section{Subscript}

$\begin{array}{ll}\text { DM } & \text { Molecular Distiller } \\ \text { F } & \text { Final Temperature } \\ \text { i } & \text { Initial Temperature }\end{array}$

\section{Appendix}

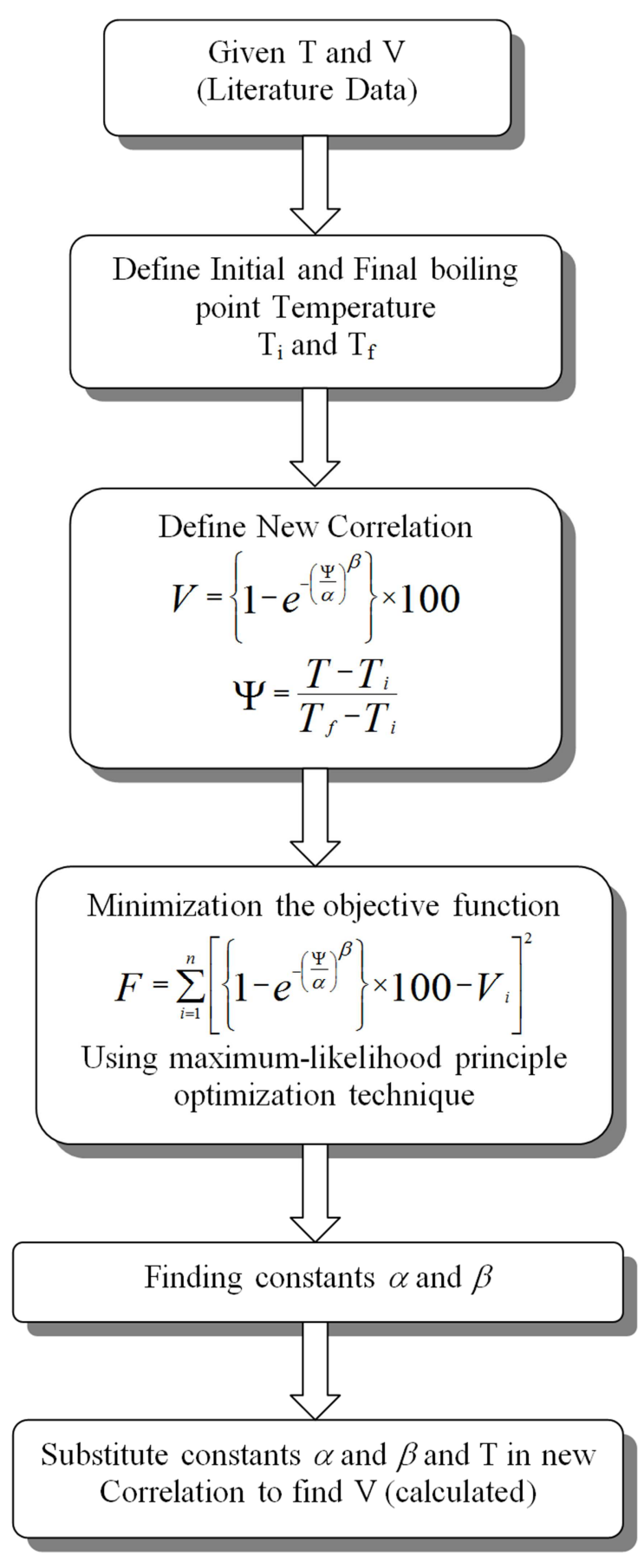

Figure 1. The flow chart of computer program. 


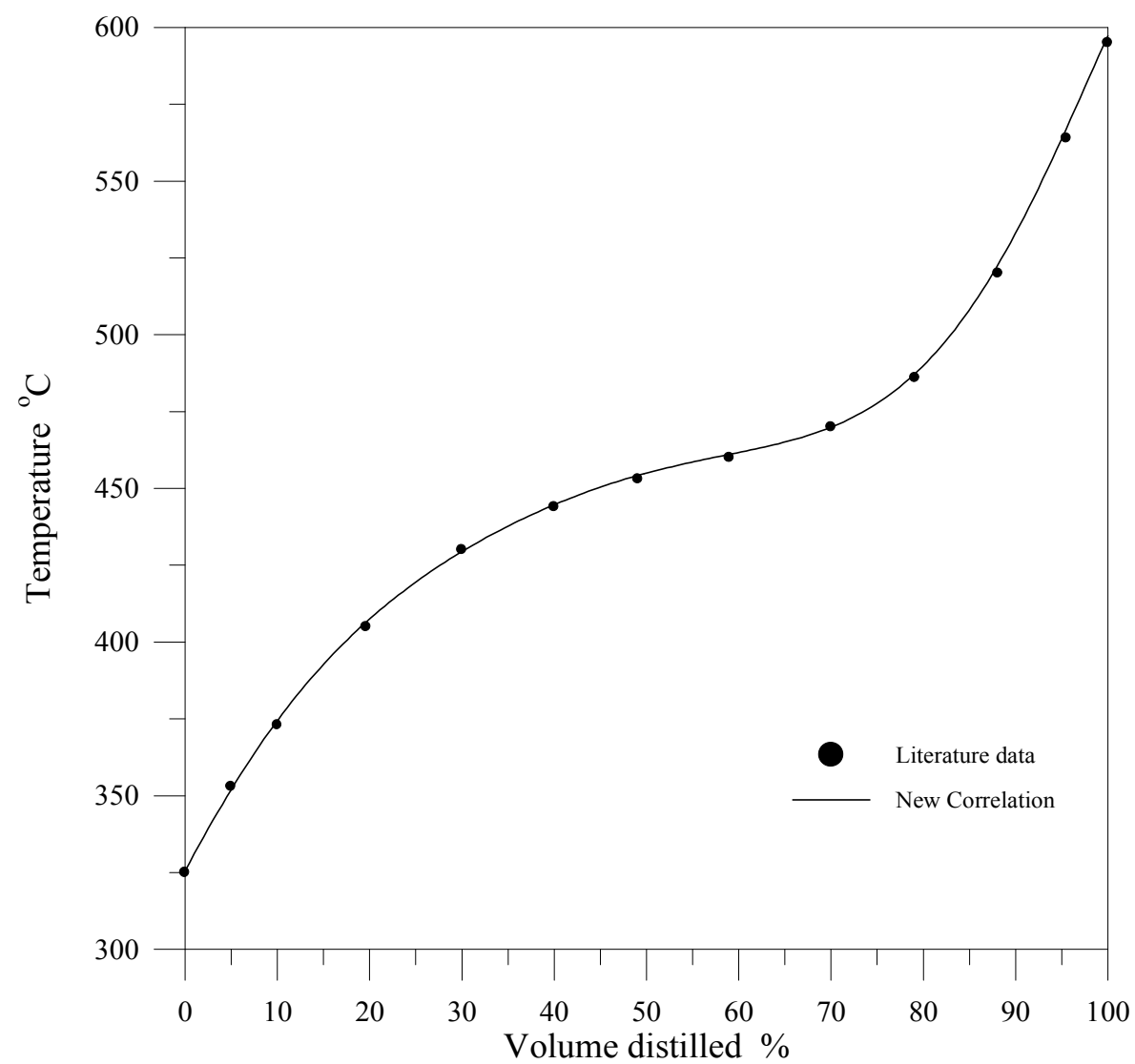

Figure 2. ASTM distillation curve for feed to FCC unit.

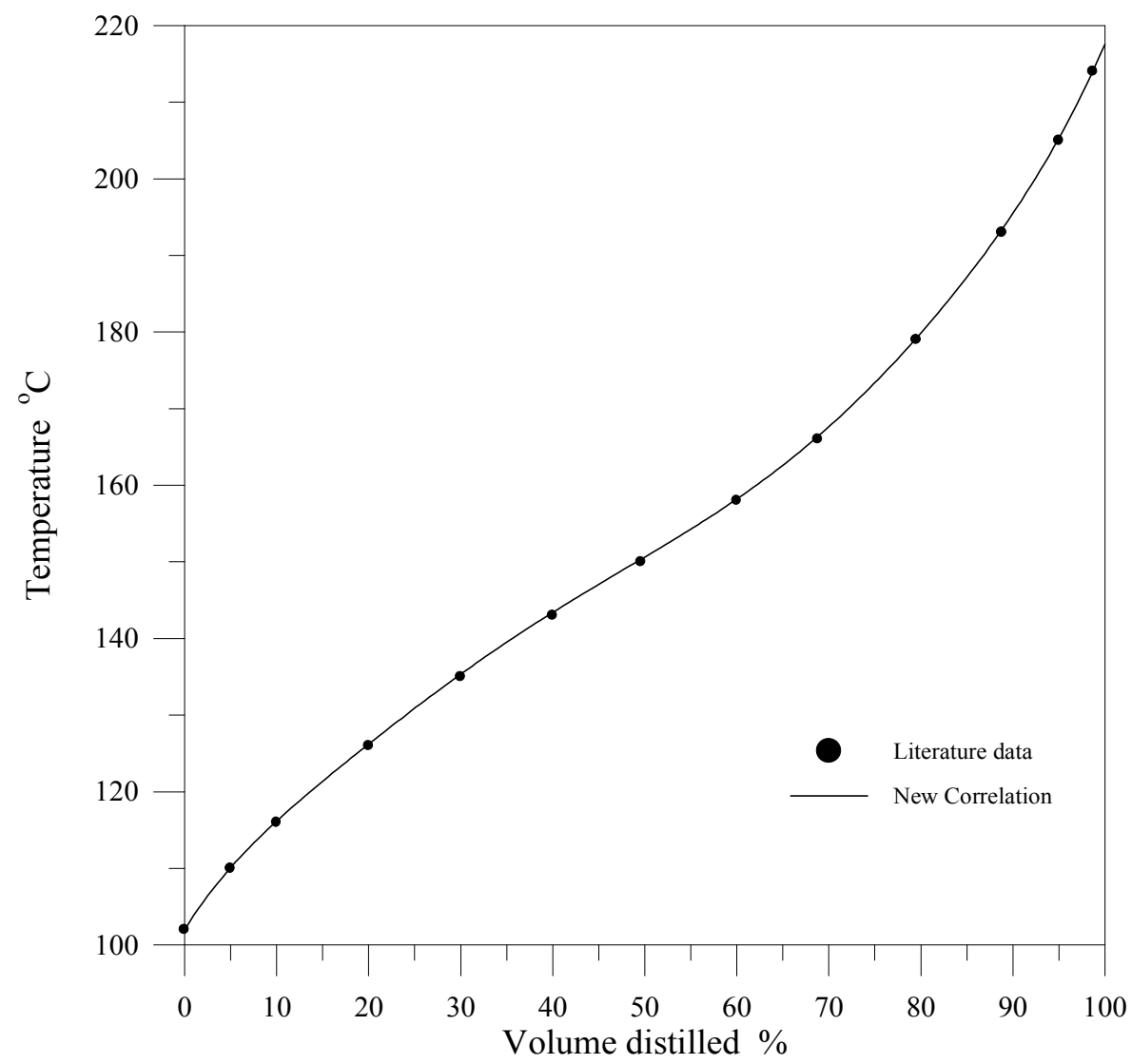

Figure 3. ASTM distillation curve for gasoline from FCC unit. 
Khalid Farhod Chasib and Srikanth Karthik P.: An Investigation on the Feasibility of Simulating the Distillation Curves and ASTM Distillation Temperature

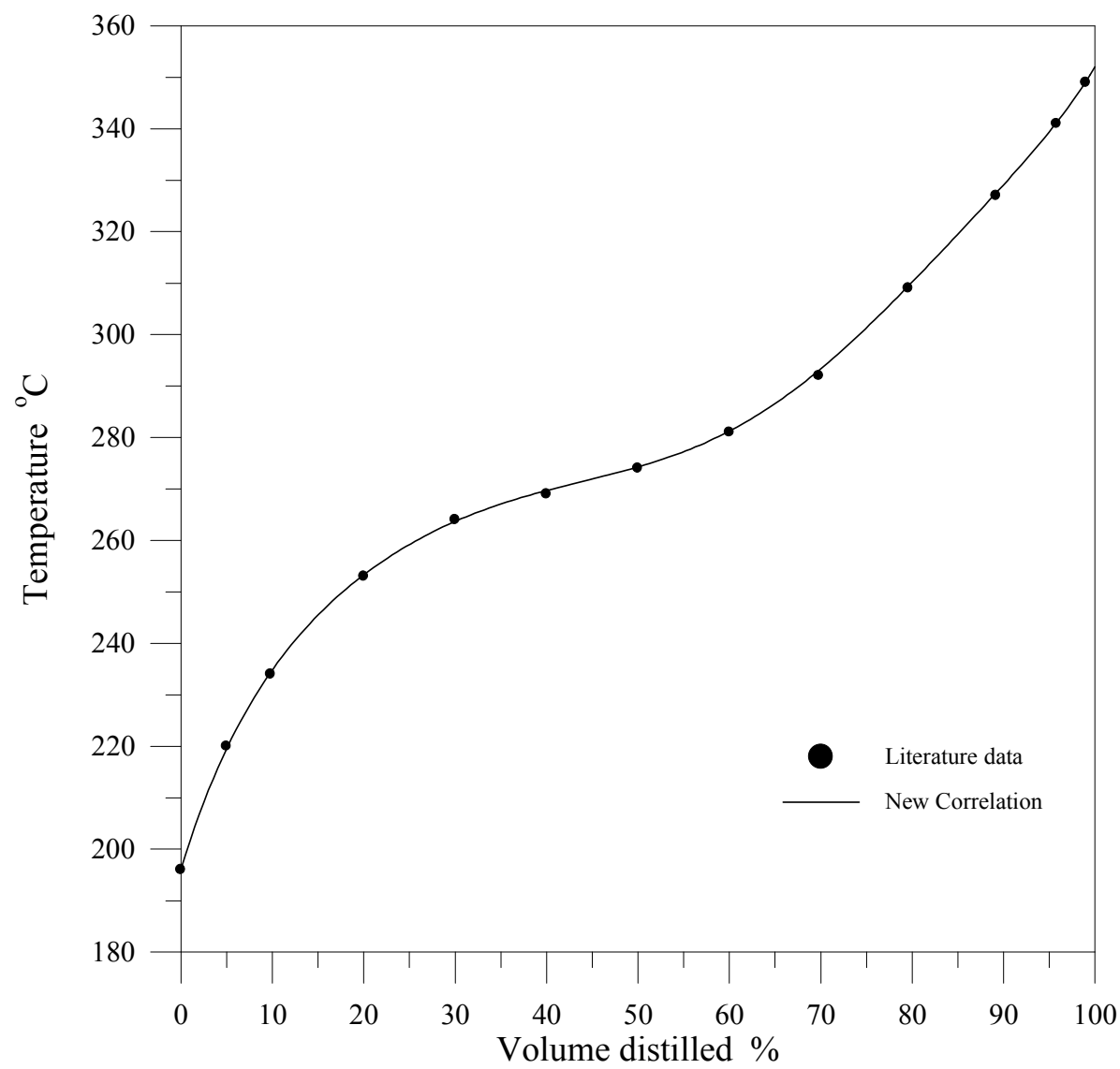

Figure 4. ASTM distillation curve for LCO from FCC unit.

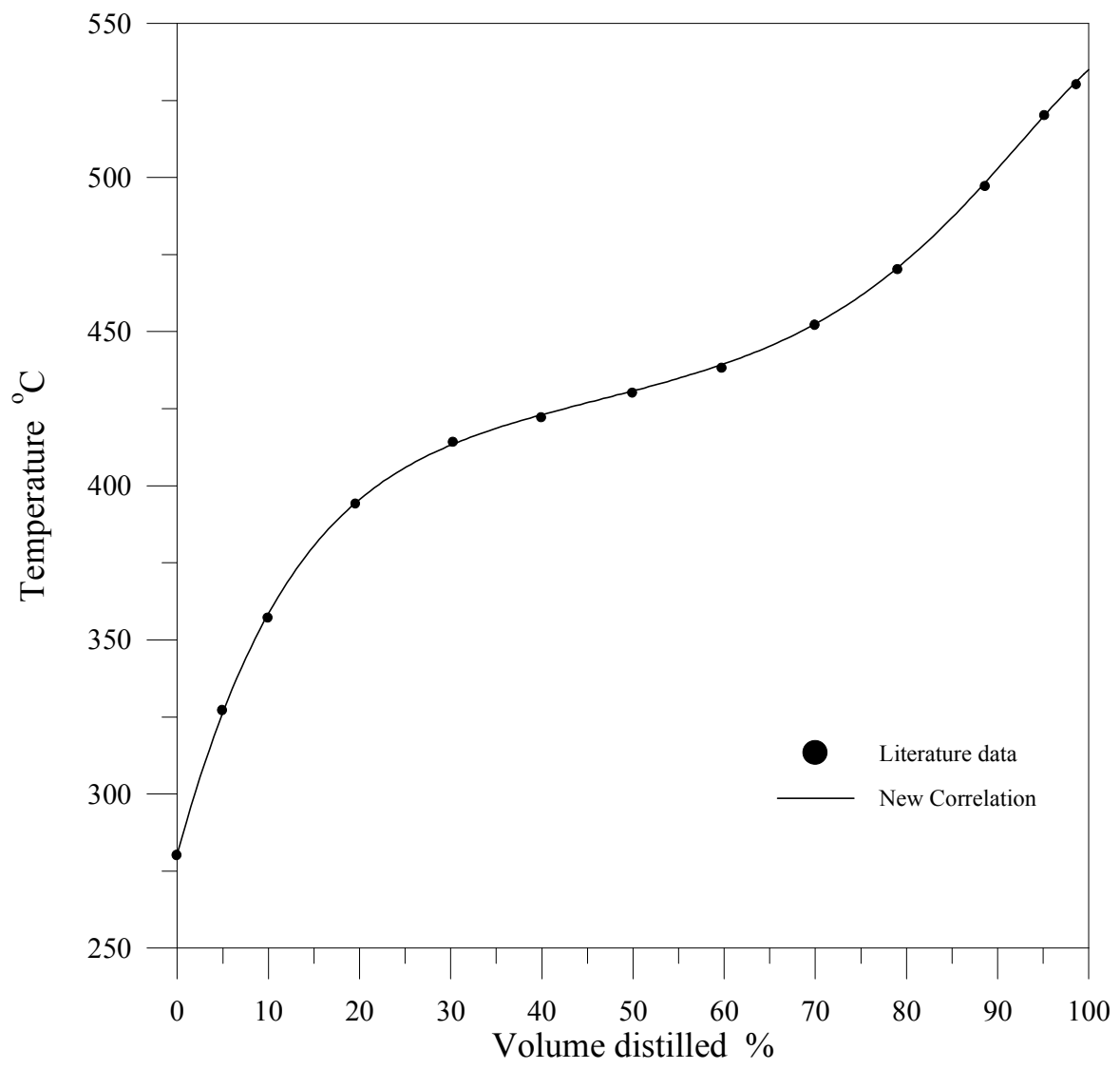

Figure 5. ASTM distillation curve for mixed product of LCO and slurry from FCC unit. 


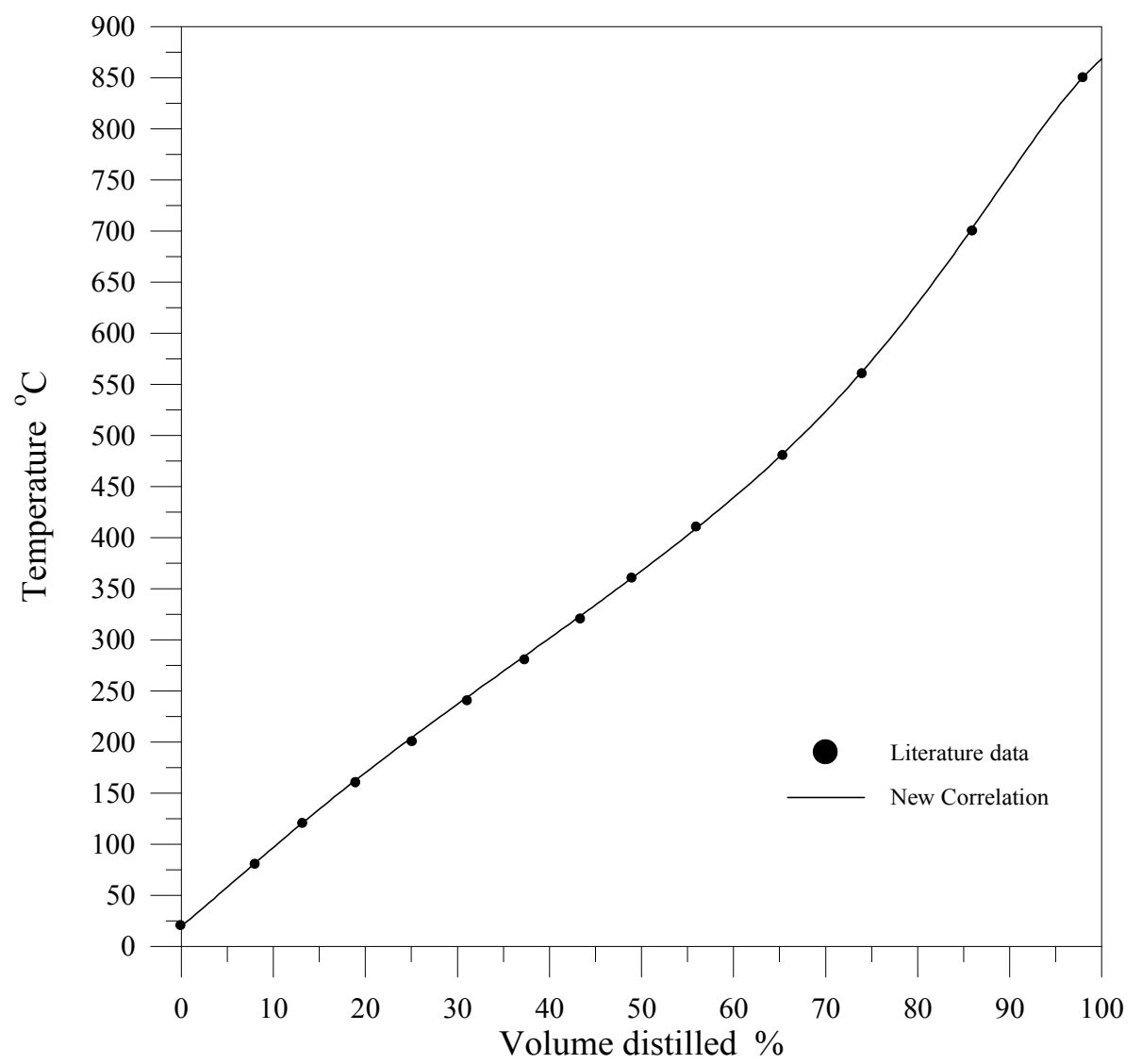

Figure 6. ASTM distillation curve for Arabian Heavy Crude Oil.

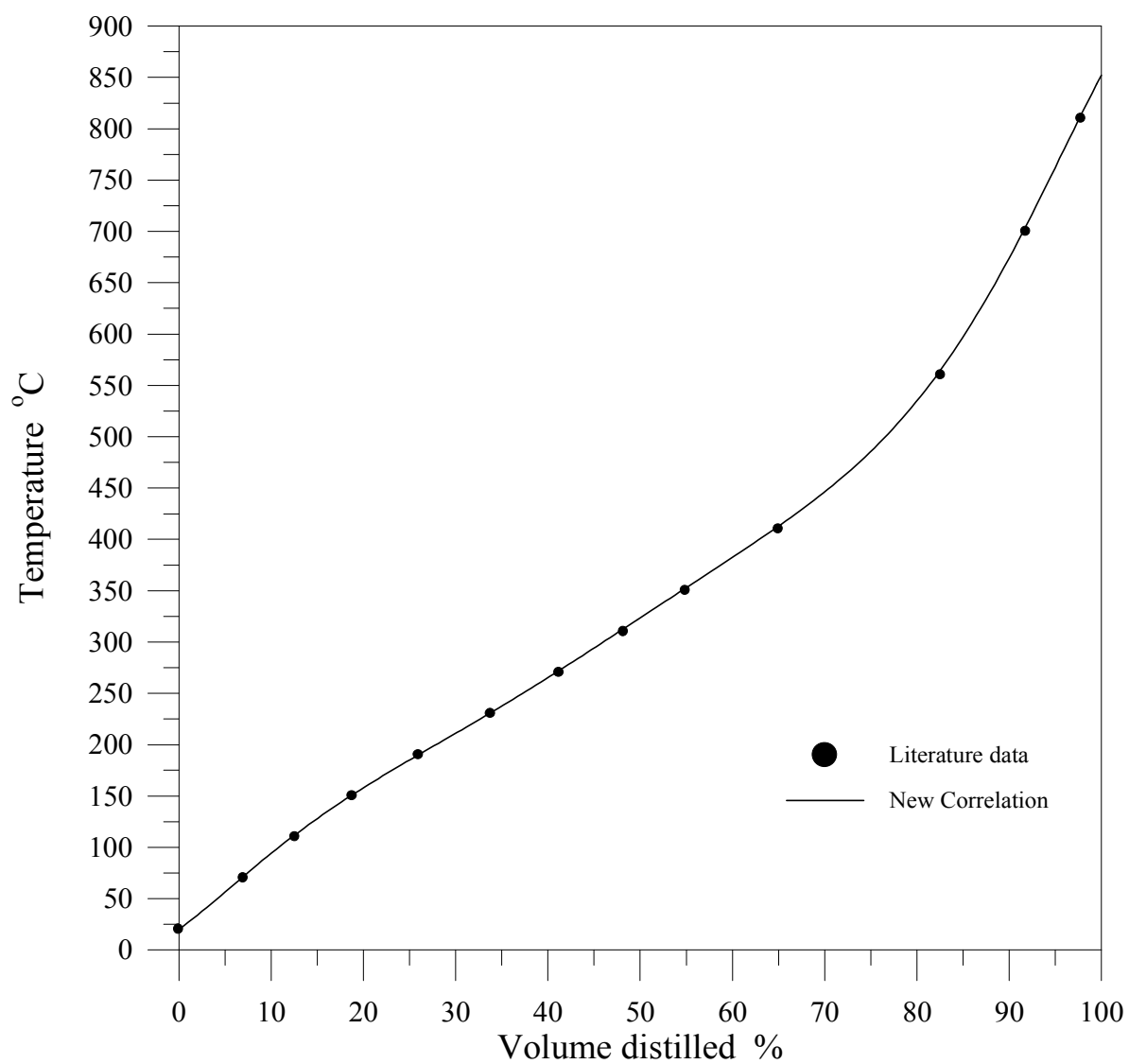

Figure 7. ASTM distillation curve for Arabian Light Crude Oil. 


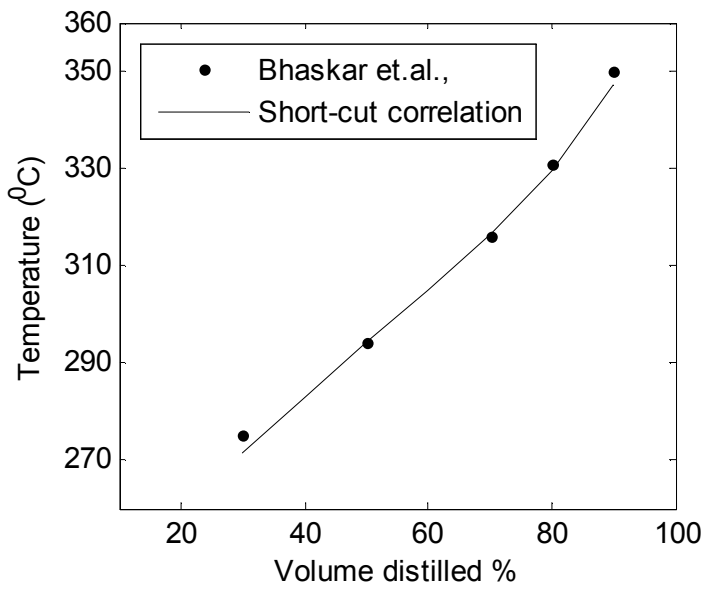

Figure 8. ASTM Distillation curve using Eq. 6 compared with data available in literature [19].

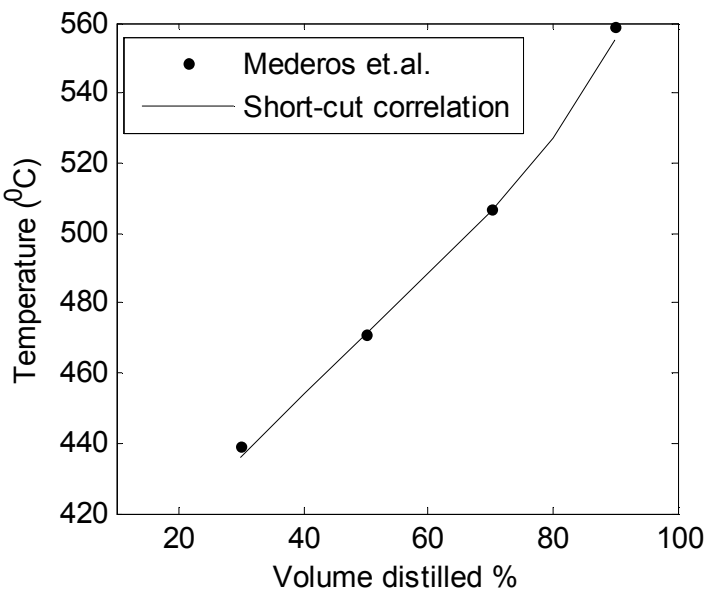

Figure 9. ASTM Distillation curve using Eq. 6 compared with data available in literature [20].

Table 1. The properties of the crude oils and petroleum fractions.

\begin{tabular}{|c|c|c|c|c|c|}
\hline Type of ASTM distillation curve & Type of crude oil or petroleum fractions & API Gravity & $T_{i},{ }^{\circ} \mathbf{C}$ & $\boldsymbol{T}_{f},{ }^{\circ} \mathrm{C}$ & Ref. No. \\
\hline Feed to FCC unit & Lindsey Oil Refinery, CFP/FINA, England & 27.6 & 325 & 595 & 2 \\
\hline Gasoline from FCC unit & Lindsey Oil Refinery, CFP/FINA, England & 16.6 & 102 & 214 & 2 \\
\hline LCO from FCC unit & Lindsey Oil Refinery, CFP/FINA, England & 15.3 & 196 & 349 & 2 \\
\hline LCO and slurry from FCC unit & Lindsey Oil Refinery, CFP/FINA, England & 9.1 & 280 & 530 & 2 \\
\hline Arabian Heavy Crude Oil & Safaniya, Saudi Arabia & 27.4 & 20 & 850 & 1 \\
\hline Arabian Light Crude Oil & Beri, Saudi Arabia & 33.4 & 20 & 810 & 1 \\
\hline
\end{tabular}

Table 2. Optimum values of constants $\alpha$ and $\beta$ and percentage of Mean Overall Deviation.

\begin{tabular}{llll}
\hline Type of ASTM distillation curve & $\alpha$ & $\beta$ & \% MOD \\
\hline Feed to FCC unit & 0.591 & 2.925 & 0.476 \\
Gasoline from FCC unit & 0.592 & 1.804 & 0.305 \\
LCO from FCC unit & 0.622 & 2.722 & 0.368 \\
LCO and slurry from FCC unit & 0.683 & 3.296 & 0.403 \\
Arabian Heavy Crude Oil & 0.601 & 1.764 & 0.585 \\
Arabian Light Crude Oil & 0.599 & 1.798 & 0.514 \\
\hline
\end{tabular}

Table 3. Universal optimum values of constants $\beta$ and $\alpha / \Psi$.

\begin{tabular}{ll}
\hline Universal Constant & Value \\
\hline$\alpha$ & 0.6 \\
$\beta$ & 3.49 \\
$\alpha / \Psi$ & 1.11 \\
\hline
\end{tabular}

\section{References}

[1] W. C. Lyons, (1996), Standard Handbook of Petroleum and Natural Gas Engineering, Gulf Publishing Company.

[2] J. G. SPEIGHT, (2002), Handbook of Petroleum Product Analysis, John Wiley \& Sons, Inc., Hoboken, New Jersey.

[3] R. J. HENGSTEBECK., (2001), PETROLEUM PROCESSING, Principles and Applications, the McGraw-Hill Book Company, Inc.

[4] L. A. Alcazar, J. Ancheyta,(2007), Sensitivity analysis based methodology to estimate the best set of parameters for heterogeneous kinetic models, Chemical Engineering Journal 12885-93.
[5] S. Sanchez, J. Ancheyta, W. C. McCaffrey,(2007), Comparison of Probability Distribution Functions for Fitting Distillation Curves of Petroleum, Energy \& Fuels, 21, 29552963.

[6] Batistella, C. B., (1999), PHD Thesis (in Portuguese), UNICAMP, SP, Brazil.

[7] Batistella, C. B. E Maciel, M. R. W., (1998), Comput. Chem. Eng., v. 22, S53-S60.

[8] Burrows, G, (1960), Molecular Distillation. Oxf. Univ. Press (Oxford).

[9] Boduszynski, M. M E Altgelt, K. H., (1994), Composition end Analysis of Heavy Petroleum Fractions. Marcel Dekker, Inc., NY.

[10] Batistella, C. B., Sbaite, P., Wolf Maciel, M. R., Maciel Filho, R., Winter, A., Gomes, A., Medina, L., Kunert, R, (2005), Heavy Petroleum Fractions Characterization: A New Approach Through Molecular Distillation, $2^{\text {nd }}$ Mercosul Congress on Chemical Engineering \& 4th Mercosul Congress on Process Systems Engineering (ENPROMER 2005), Costa Verde - RJ, Brazil.

[11] Winter, A., Batistella, C. B., Wolf Maciel, M. R., Maciel Filho, R., Lopes, M. S. Medina, L. C., A True Boiling Point Curve Through Molecular Distillation Using FRAMOL Correlation, Internet resources available at (http://www.nt.ntnu.no/users/skoge/prost/proceedings/icheap8 -pres07/icheap8webpapers/109\%20Winter.pdf)

[12] Randy B., Ian B., (2006), Sensitivity of Oil Fate Model Predictions to Oil Property Inputs, Can. J Chem. Engng, 83, 3.

[13] Owen, K. and Coley, T., (1995), "Automotive Fuels Reference Book", $2^{\text {nd }}$ ed., Warrendale, PA. Society of Automotive Engineers. 
[14] P. Englezoz and N. Kalogerakis, (2001), Applied Parameters Estimation for Chemical Engineers, Marcel Dekker, INC.

[15] Anderson, T. F., Abrams, D. S., Grens, E. A., (1978), "Evaluation of parameters for Nonlinear Thermodynamic Models", AIChE J., 24, 20, pp 16-21.

[16] Jones D. S. D. and Pujado P. R., (2006). Handbook of Petroleum Processing, Springer, Netherlands.

[17] Maples R. E., (2000). Petroleum Refinery Process Economics, 2nd Edition, Pennwell Corporation, USA.
[18] Technical Data book - Petroleum Refining (1997), 6th Edition, American Petroleum Institute (API).

[19] Bhaskar, M., Valavarasu, G., Sairam, B., Balaraman, K. S., Balu, K., 2004. Industrial \&Engineering Chemistry Research 43, 6654-6669.

[20] Khalid F. C., (2013), Study on the effect of adding co-solvent (n-alkoxyethanol) to sulfolane on the toluene extraction, Scientia Iranica C, 20, 1899-1911.

[21] Mederos, F. S., Rodríguez, M. A., Ancheyta, J., Arce, E., 2006. Energy Fuels 20, 936-945. 